\title{
The First Practical Additive-Free 1,4-Conjugated Alkylation of Fluoro- alkylated Electron-Deficient Olefins with Various Organozinc Reagents
}

\author{
Atsunori Morigaki, ${ }^{a}$ Tomotsugu Miyabe, ${ }^{b}$ Kazuki Tsukade, ${ }^{b}$ Satoru Arimitsu, ${ }^{\mathrm{c}}$ Takashi Ishihara, ${ }^{\mathrm{b}}$ Tsutomu Konno*b \\ a Functional Materials Research Laboratories Research \& Development Headquarters LION CORPORATION, 7-2-1 Hirai, Edogawa-ku, \\ Tokyo 132-0035, Japan \\ b Department of Chemistry and Materials Technology, Kyoto Institute of Technology, Matsugasaki, Sakyo-ku, Kyoto 606-0962, Japan \\ Fax +81(75)7247580; E-mail: konno@chem.kit.ac.jp \\ c Department of Chemistry Biology and Marine Science, University of the Ryukyus, Nishihara, Okinawa 903-0123, Japan \\ Received: 30.09.2012; Accepted after revision: 08.11.2012
}

\begin{abstract}
Conjugated alkylation of fluoroalkylated olefins with organozinc reagents, such as $\mathrm{RZnI}$ and $\mathrm{R}_{2} \mathrm{Zn}$, was conducted smoothly to give the corresponding products in moderate yields without the use of either transition metals or Lewis acids. This reaction protocol allows not only a wide range of alkyl groups but also electron-withdrawing groups to be incorporated as 1,4-conjugated adducts.
\end{abstract}

Key words: 1,4-conjugated alkylation, organozinc reagents, trifluoromethyl, electron-deficient olefin

The carbon-carbon bond formation through 1,4-conjugated addition has been recognized as one of the most important organic reactions. In the last decade, numerous synthetic methodologies have been developed for this transformation. ${ }^{1}$ Especially the transition-metal-catalyzed 1,4-conjugated addition has been intensively investigated and applied for enantioselective versions in several successful examples. ${ }^{2}$ Among these developments, the metal nucleophile also has been studied to search for expanding substrate scopes under the milder reaction condition. The organozinc reagents (i.e., $\mathrm{RZnX}, \mathrm{R}_{2} \mathrm{Zn}$, and $\mathrm{R}_{3} \mathrm{ZnMet}$ ) are known as unique nucleophiles compared to other common organometallic reagents, that is, the softer nucleophilicity of organozinc reagents shows higher functional-group tolerance. ${ }^{3}$ However, organozinc reagents often require the assistance of transition metals or Lewis acids in order to react as expected. ${ }^{4}$

Tremendous attention has been paid to fluorine-containing molecules because of their biological properties as well as their unique reactivities, which has led to new developments of synthetic methodologies. ${ }^{5}$ For example, trifluoromethyl-bearing olefins, such as $\mathbf{1}$ in Scheme 1, shows very interesting LUMO-lowering effect on its $\beta$-carbon. ${ }^{6}$ We and Yamazaki et al. ${ }^{7}$ have demonstrated that the electron-deficient olefin $\mathbf{1}$ can serve as an excellent reaction partner for 1,4-conjugated addition, but incoming substrates for these reactions are to some extent limited. In fact, the introduction of simple alkyl substrates has been facing many difficulties, such as defluorination and a nar-

SYNTHESIS 2013, 45, 0101-0105

Advanced online publication: 27.11.2012

DOI: 10.1055/s-0032-1317707; Art ID: SS-2012-F0734-OP

(C) Georg Thieme Verlag Stuttgart · New York row range of the substrate scope. ${ }^{7}$ To overcome this limitation, we revisited the basic nature of olefin $\mathbf{1}$, and realized that it will be possible for even unreactive nucleophiles to react with olefin 1 without addition of any activators (i.e., transition metals and Lewis acids). Herein, we describe the first practical 1,4-conjugated alkylation of fluoroalkylated olefins 1 with organozinc reagents under the additive-free conditions.

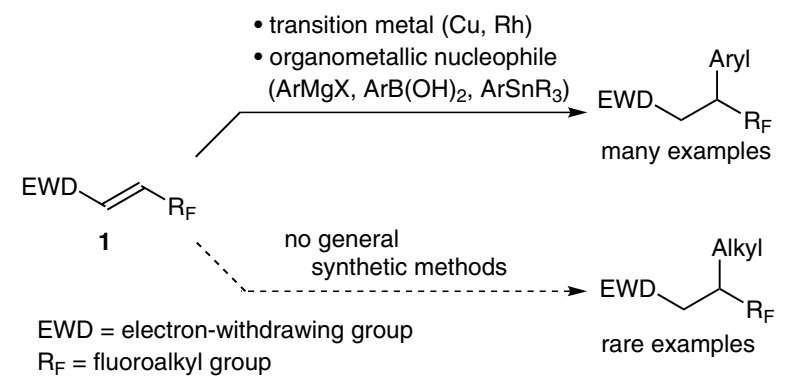

Scheme 1 1,4-Conjugated addition to fluoroalkylated electrondeficient olefins 1 with organometallic reagents

Initially, the least reactive organozinc reagents, alkylzinc halides, were chosen for the 1,4-conjugated alkylation to (E)-4,4,4-trifluoro-1-phenylbut-2-en-1-one (1a). Thus, the olefin 1a was treated with 3.0 equivalents of freshly prepared $\mathrm{RZnI}^{8}$ in THF at $0{ }^{\circ} \mathrm{C}$ and then the reaction mixture was stirred for eight hours at the same temperature. To our surprise, the reaction gave the corresponding 1,4adduct in a moderate yield without extra additive (Table 1 , entries 1 and 3). Next, the same reaction was tested with dialkylzinc reagents, ${ }^{9}$ which gave a smoother reaction and higher yield of the product in only one hour at $-78^{\circ} \mathrm{C}$ (entries 2 and 4). Interestingly, when the organozincate, $(n-\mathrm{Bu})_{3} \mathrm{ZnLi},{ }^{10}$ was utilized in this reaction, its 1,2 -adduct was obtained instead as the major product (entry 5). This reaction trend of organozinc reagents was observed similarly in other substrates; therefore, $\mathrm{RZnI}$ and $\mathrm{R}_{2} \mathrm{Zn}$ were used for further reactions. Dialkylzinc reagents were found to be the most effective, ${ }^{11}$ and even sterically hindered alkyl groups, such as $i$-Pr and $t$-Bu, could be introduced in moderate yields (entries 8 and 9). However, in contrast by taking full advantage of the mild reactivity of organozinc halides, the alkyl groups with labile function- 
alities could also be installed into the desired structure (entries 10 and 11).

Table 1 1,4-Conjugated Alkylation of Olefin 1a with Various Organozinc Reagents<smiles>O=C(/C=C/C(F)(F)F)c1ccccc1</smiles>

1a

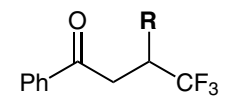

\begin{tabular}{lllllll}
\hline Entry & $\begin{array}{l}\text { Organozinc } \\
\text { reagent }\end{array}$ & Equiv & $\begin{array}{c}\text { Method }^{\mathrm{a}} \\
(\mathrm{h})\end{array}$ & $\begin{array}{c}\text { Time } \\
\text { Product }\end{array}$ & $\begin{array}{l}\text { Yield } \\
(\%)^{\mathrm{b}}\end{array}$ \\
\hline 1 & $\mathrm{EtZnI}$ & 3.0 & $\mathrm{~A}$ & 8 & $\mathbf{2 a a}$ & $57(54)$ \\
2 & $\mathrm{Et}_{2} \mathrm{Zn}^{\mathrm{c}}$ & 1.2 & $\mathrm{~B}$ & 1 & $\mathbf{2 a a}$ & $83(82)$ \\
3 & $n-\mathrm{BuZnI}$ & 3.0 & $\mathrm{~A}$ & 8 & $\mathbf{2 a b}$ & $62(37)$ \\
4 & $(n-\mathrm{Bu})_{2} \mathrm{Zn}$ & 2.4 & $\mathrm{~B}$ & 2 & $\mathbf{2 a b}$ & $81(56)$ \\
5 & $(n-\mathrm{Bu})_{3} \mathrm{ZnLi}$ & 3.0 & $\mathrm{C}$ & 2 & $\mathbf{2 a b}$ & $27^{\mathrm{d}}$ \\
6 & $(n-\mathrm{Hex})_{2} \mathrm{Zn}$ & 2.4 & $\mathrm{~B}$ & 2 & $\mathbf{2 a c}$ & $66(61)$ \\
7 & $c-\mathrm{HexZnI}$ & 2.4 & $\mathrm{~A}$ & 8 & $\mathbf{2 a d}$ & $50^{\mathrm{e}}$ \\
8 & $(i-\mathrm{Pr})_{2} \mathrm{Zn}{ }^{\mathrm{c}}$ & 1.2 & $\mathrm{~B}$ & 1 & $\mathbf{2 a e}$ & $86(62)$ \\
9 & $(t-\mathrm{Bu})_{2} \mathrm{Zn}$ & 2.4 & $\mathrm{~B}$ & 2 & $\mathbf{2 a f}$ & $50^{\mathrm{e}}$ \\
10 & $\mathrm{EtO}_{2} \mathrm{C}(\mathrm{CH})_{3} \mathrm{ZnI}$ & 3.0 & $\mathrm{~A}$ & 8 & $\mathbf{2 a g}$ & $54^{\mathrm{e}}$ \\
11 & $\mathrm{NC}\left(\mathrm{CH}_{2}\right)_{3} \mathrm{ZnI}$ & 3.0 & $\mathrm{~A}$ & 8 & $\mathbf{2 a h}$ & $49^{\mathrm{e}}$ \\
\hline
\end{tabular}

${ }^{\text {a }}$ Method A: in THF $(0.25 \mathrm{M})$ at $0{ }^{\circ} \mathrm{C}$. Method B: in toluene $(0.125 \mathrm{M})$ at $-78^{\circ} \mathrm{C}$. Method $\mathrm{C}$ : in THF $(0.25 \mathrm{M})$ at $-78^{\circ} \mathrm{C}$.

${ }^{\mathrm{b}}$ Yields were determined by ${ }^{19} \mathrm{~F}$ NMR spectroscopy, and the values in parentheses are isolated yields.

${ }^{\mathrm{c}}$ The commercially available salt-free organozinc reagents were used.

$\mathrm{d}$ The 1,2-adduct was isolated as the major product in $69 \%$.

${ }^{\mathrm{e}}$ The pure products were inseparable from impurities.

Based on the known reactivity of organozinc reagents, $\mathrm{Et}_{2} \mathrm{Zn}$ was chosen for the 1,4-conjugated alkylation with various types of electron-deficient olefins, and these results are shown in Table 2. Although slight modifications were required from the original condition depending on the reactivity of olefins $\mathbf{1}$, most electron-withdrawing groups (EWDs) can survive under the given reaction condition without formation of any noticeable by-products (Table 2, entries 1-4). The olefin with a difluoromethyl $\left(\mathrm{CF}_{2} \mathrm{H}\right)$ group also reacted smoothly to give the product in excellent yield (entry 5 ).

Finally, preliminary work on the possibility of diastereoselective 1,4-conjugated alkylation using trifluoromethylated alkenes with Evan's chiral auxiliary was conducted (Scheme 2). Thus, treatment of $\mathbf{1 f}$ or $\mathbf{1 g}$ with 3.6 equivalents of $\mathrm{Et}_{2} \mathrm{Zn}$ at $-40{ }^{\circ} \mathrm{C}$ for 24 hours gave the corresponding 1,4-adducts in good yields; however, as diastereomeric mixtures in both cases. Further effort to attain higher diastereoselectivity is currently underway in our laboratory.
Table 2 1,4-Conjugated Alkylation of Various Fluoroalkylated Electron-Deficient Olefins 1 with $\mathrm{Et}_{2} \mathrm{Zn}^{\mathrm{a}}$

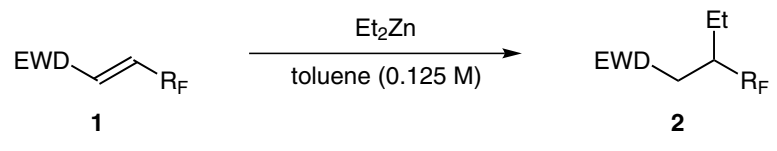

\begin{tabular}{lllllll}
\hline Entry & $\mathrm{EWD}$ & $\mathrm{R}_{\mathrm{F}}$ & $\begin{array}{l}\mathrm{Et}_{2} \mathrm{Zn} \\
\text { (equiv) }\end{array}$ & $\begin{array}{l}\mathrm{Temp} \\
\left({ }^{\circ} \mathrm{C}\right)\end{array}$ & Product & $\begin{array}{l}\text { Yield } \\
(\%)^{\mathrm{b}}\end{array}$ \\
\hline $1^{\mathrm{c}}$ & $\mathrm{C}(\mathrm{O}) \mathrm{Ph}$ & $\mathrm{CF}_{3}$ & 1.2 & -78 & $\mathbf{2 a a}$ & $83(82)$ \\
$2^{\mathrm{d}}$ & $\mathrm{C}(\mathrm{O}) \mathrm{NBn}_{2}$ & $\mathrm{CF}_{3}$ & 3.6 & -40 & $\mathbf{2 b a}$ & $80(74)$ \\
$3^{\mathrm{d}}$ & $\mathrm{SO}_{2} \mathrm{Ph}$ & $\mathrm{CF}_{3}$ & 3.6 & -20 & $\mathbf{2 c a}$ & $75(68)$ \\
$4^{\mathrm{d}}$ & $\mathrm{P}(\mathrm{O})(\mathrm{OEt})_{2}$ & $\mathrm{CF}_{3}$ & 3.6 & -20 & $\mathbf{2 d a}$ & $72(69)$ \\
$5^{\mathrm{c}}$ & $\mathrm{C}(\mathrm{O}) \mathrm{Ph}$ & $\mathrm{CF}_{2} \mathrm{H}$ & 2.4 & -78 & $\mathbf{2 e a}$ & $92(85)$
\end{tabular}

a The reaction was conducted following method B of Table 1 but at different temperatures.

${ }^{\mathrm{b}}$ Yields were determined by ${ }^{19} \mathrm{~F}$ NMR spectroscopy, and the values in parentheses are isolated yields.

${ }^{\mathrm{c}}$ The reaction time was $1 \mathrm{~h}$.

${ }^{\mathrm{d}}$ The reaction time was $24 \mathrm{~h}$.

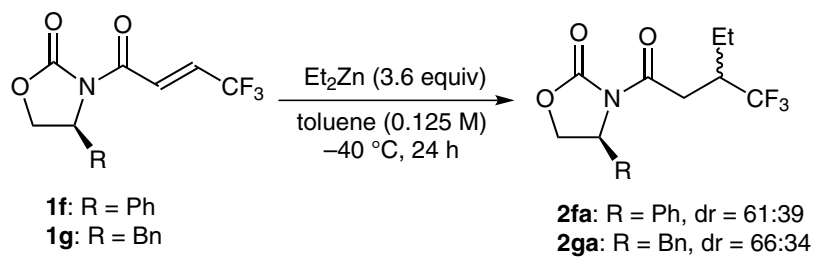

Scheme 2 Preliminary results of the conjugated addition using chiral substrates

In summary, we have examined the simple yet practical 1,4-conjugated alkylation of fluoroalkylated electrondeficient olefins with various unreactive organozinc reagents without any additives. ${ }^{12}$ As a result, this reaction protocol can allow many labile alkyl groups to participate in 1,4-conjugated addition reaction; that is, our new methodology can compensate for the previously established 1,4-conjugated addition limited strictly to aromatic nucleophiles.

${ }^{1} \mathrm{H}$ and ${ }^{13} \mathrm{C}$ NMR spectra were recorded on a Bruker DRX-500 $\left(500.13 \mathrm{MHz}\right.$ for ${ }^{1} \mathrm{H}$ and $125.75 \mathrm{MHz}$ for ${ }^{13} \mathrm{C}$ ) spectrometer on samples dissolved in $\mathrm{CDCl}_{3}$ with $\mathrm{Me}_{4} \mathrm{Si}$ as an internal reference. A Jeol JNM-AL400 (376.05 MHz) spectrometer was used to record ${ }^{19} \mathrm{~F}$ NMR spectra in $\mathrm{CDCl}_{3}$ using $\mathrm{CFCl}_{3}$ as an internal standard. IR spectra were recorded as liquid film or $\mathrm{KBr}$ disk with an Avtar370DTGS spectrometer (Thermo Electron) or an FT/IR-4100 (JASCO) spectrometer. High-resolution mass spectra were taken with a JEOL JMS-700 MS spectrometer. Column chromatography was carried out on silica gel (Wako gel C-200) and TLC analysis was performed on silica gel TLC plates (Merck, Silica gel $60 \mathrm{~F}_{254}$ ). All reactions were carried out under an atmosphere of argon. Anhyd THF and $\mathrm{Et}_{2} \mathrm{O}$ were purchased from Wako Pure Chemical Industries, Ltd. $n$-BuLi (1.6 M hexane solution) was commercially available from Wako Pure Chemical Industries, Ltd. All chemicals were of reagent grade and, if necessary, were purified in the usual manner prior to use. 
1,4-Conjugated Alkylation of RZnI to 4,4,4-Trifluoro-1-phenylbut-2-enone (1a); General Procedure for Method A

To a solution of 4,4,4-trifluoro-1-phenylbut-2-enone $(\mathbf{1 a} ; 50 \mathrm{mg}$, $0.25 \mathrm{mmol})$ in THF $(1 \mathrm{~mL})$ was added alkylzinc iodide (freshly prepared prior to the reaction from RI and $\mathrm{Zn}$ dust $^{8}$ ) in THF at $0{ }^{\circ} \mathrm{C}$. The mixture was then stirred for $8 \mathrm{~h}$ at $0{ }^{\circ} \mathrm{C}$ and quenched with sat. aq $\mathrm{NH}_{4} \mathrm{Cl}(5 \mathrm{~mL})$. The mixture was extracted with EtOAc $(3 \times 10$ $\mathrm{mL})$, and the combined organic layers were dried $\left(\mathrm{Na}_{2} \mathrm{SO}_{4}\right)$. The organic solvents were removed, and the residue was purified by silica gel column chromatography to give the corresponding product.

1,4-Conjugated Alkylation of $R_{2} \mathrm{Zn}$ to 4,4,4-Trifluoro-1-phenylbut-2-enone (1a); General Procedure for Method $B$

To a solution of 4,4,4-trifluoro-1-phenylbut-2-enone $(\mathbf{1 a} ; 50 \mathrm{mg}$, $0.25 \mathrm{mmol})$ in toluene $(2 \mathrm{~mL})$ was added a $1.0 \mathrm{M}$ hexane solution of $\mathrm{R}_{2} \mathrm{Zn}\left(0.3 \mathrm{~mL}, 1.2\right.$ equiv) [in case of in situ generation of $\mathrm{R}_{2} \mathrm{Zn}$; $0.6 \mathrm{~mL}$ of $\mathrm{ZnCl}_{2}\left(1.0 \mathrm{M}, \mathrm{Et}_{2} \mathrm{O}\right.$ solution) was added to $0.75 \mathrm{~mL}$ of $\operatorname{RLi}\left(1.6 \mathrm{M}\right.$, hexane solution) at $0^{\circ} \mathrm{C}$, and stirred for $30 \mathrm{~min}$ at $\left.0{ }^{\circ} \mathrm{C}\right]$ at $-78^{\circ} \mathrm{C}$, and stirred for $1 \mathrm{~h}$ at $-78^{\circ} \mathrm{C}$. The reaction was quenched with sat. aq $\mathrm{NH}_{4} \mathrm{Cl}(10 \mathrm{~mL})$, extracted with EtOAc $(3 \times 10 \mathrm{~mL})$, and the combined organic layers were dried $\left(\mathrm{Na}_{2} \mathrm{SO}_{4}\right)$. The organic solvents were removed, and the residue was purified by silica gel column chromatography to give the corresponding product.

\section{1-Phenyl-3-trifluoromethylpentan-1-one (2aa)}

Method B; yield: $47 \mathrm{mg}(0.21 \mathrm{mmol}, 82 \%)$; yellow oil.

IR (neat): 3063, 2973, 2943, 2886, 1691, 1598, 1582, 1465, 1450, $1422,1394,1349,1326,1307,1257,1219 \mathrm{~cm}^{-1}$.

${ }^{1} \mathrm{H} \mathrm{NMR}\left(\mathrm{CDCl}_{3}\right): \delta=1.00(\mathrm{t}, J=7.5 \mathrm{~Hz}, 3 \mathrm{H}), 1.49-1.59(\mathrm{~m}, 1 \mathrm{H})$, 1.73-1.81 (m, 1 H), 2.97-3.08 (m, $2 \mathrm{H}), 3.22-3.28(\mathrm{~m}, 1 \mathrm{H}), 7.45-$ 7.51 (m, 2 H), 7.57-7.61 (m, $1 \mathrm{H}), 7.96-7.98$ (m, $2 \mathrm{H})$.

${ }^{13} \mathrm{C} \mathrm{NMR}\left(\mathrm{CDCl}_{3}\right): \delta=11.25,21.73,36.73(\mathrm{q}, J=2.5 \mathrm{~Hz}), 39.43(\mathrm{q}$, $J=25.8 \mathrm{~Hz}), 128.03,128.37(\mathrm{q}, J=279.6 \mathrm{~Hz}), 128.73,133.45$, $136.49,196.50$.

${ }^{19} \mathrm{~F} \mathrm{NMR}\left(\mathrm{CDCl}_{3}\right): \delta=-71.09(\mathrm{~d}, J=9.8 \mathrm{~Hz}, 3 \mathrm{~F})$.

HRMS: $m / z$ calcd for $\mathrm{C}_{12} \mathrm{H}_{13} \mathrm{~F}_{3} \mathrm{O}\left(\mathrm{M}^{+}\right)$: 230.0918; found: 230.0913 .

\section{1-Phenyl-3-trifluoromethylheptan-1-one (2ab)}

Method B; yield: $36 \mathrm{mg}$ (0.14 mmol, 56\%); yellow oil.

IR (neat): 3063, 2959, 2874, 1692, 1598, 1582, 1450, 1421, 1395, $1352,1328,1268,1221,1165,1130,1096 \mathrm{~cm}^{-1}$.

${ }^{1} \mathrm{H} \mathrm{NMR}\left(\mathrm{CDCl}_{3}\right): \delta=0.89(\mathrm{t}, J=7.2 \mathrm{~Hz}, 3 \mathrm{H}), 1.27-1.40(\mathrm{~m}, 4 \mathrm{H})$ 1.42-1.49 (m, 1 H), 1.69-1.76 (m, $1 \mathrm{H}), 3.01-3.13(\mathrm{~m}, 1 \mathrm{H}), 3.03$ $(\mathrm{dd}, J=17.2,7.1 \mathrm{~Hz}, 1 \mathrm{H}), 3.27$ (dd, $J=17.2,3.6 \mathrm{~Hz}, 1 \mathrm{H}), 7.46$ 7.51 (m, 2 H), 7.57-7.61 (m, 1 H), 7.95-7.98 (m, 2 H).

${ }^{13} \mathrm{C} \mathrm{NMR}\left(\mathrm{CDCl}_{3}\right): \delta=13.71,22.60,28.50(\mathrm{q}, J=1.8 \mathrm{~Hz}), 28.89$, $37.23(\mathrm{q}, J=2.5 \mathrm{~Hz}), 38.08(\mathrm{q}, J=26.1 \mathrm{~Hz}), 128.02,128.70,128.37$ (q, $J=279.6 \mathrm{~Hz}), 133.41,136.49,196.44$.

${ }^{19} \mathrm{~F} \mathrm{NMR}\left(\mathrm{CDCl}_{3}\right): \delta=-71.32(\mathrm{~d}, J=9.8 \mathrm{~Hz}, 3 \mathrm{~F})$.

HRMS: $m / z$ calcd for $\mathrm{C}_{14} \mathrm{H}_{18} \mathrm{~F}_{3} \mathrm{O}(\mathrm{M}+\mathrm{H})$ : 259.1310; found: 259.1304 .

\section{1-Phenyl-3-trifluoromethylnonan-1-one (2ac)}

Method B; yield: $44 \mathrm{mg}(0.15 \mathrm{mmol}, 61 \%)$; yellow oil.

IR (neat): 3063, 2931, 2860, 1745, 1692, 1598, 1582, 1450, 1421, $1351,1255,1218,1163,1131,1100,1002 \mathrm{~cm}^{-1}$.

${ }^{1} \mathrm{H} \mathrm{NMR}\left(\mathrm{CDCl}_{3}\right): \delta=0.87(\mathrm{t}, J=6.9 \mathrm{~Hz}, 3 \mathrm{H}), 1.21-1.48(\mathrm{~m}, 9 \mathrm{H})$, $1.68-1.75(\mathrm{~m}, 1 \mathrm{H}), 3.00-3.12(\mathrm{~m}, 1 \mathrm{H}), 3.02(\mathrm{dd}, J=17.1,7.3 \mathrm{~Hz}$, $1 \mathrm{H}), 3.27$ (dd, $J=17.1,3.6 \mathrm{~Hz}, 1 \mathrm{H}), 7.46-7.51(\mathrm{~m}, 2 \mathrm{H}), 7.57-7.61$ (m, $1 \mathrm{H}), 7.95-7.98(\mathrm{~m}, 2 \mathrm{H})$.

${ }^{13} \mathrm{C} \mathrm{NMR}\left(\mathrm{CDCl}_{3}\right): \delta=13.99,22.52,26.74,28.83(\mathrm{q}, J=1.8 \mathrm{~Hz})$, $29.22,31.51,37.27(\mathrm{q}, J=2.1 \mathrm{~Hz}), 38.17$ (q, $J=26.1 \mathrm{~Hz}), 128.05$, 128.37 (q, $J=279.6 \mathrm{~Hz}), 128.73,133.44,136.52,196.52$.

${ }^{19} \mathrm{~F} \mathrm{NMR}\left(\mathrm{CDCl}_{3}\right): \delta=-71.31(\mathrm{~d}, J=7.1 \mathrm{~Hz}, 3 \mathrm{~F})$.
HRMS: $m / z$ calcd for $\mathrm{C}_{16} \mathrm{H}_{22} \mathrm{~F}_{3} \mathrm{O}(\mathrm{M}+\mathrm{H})$ : 287.1623; found: 287.1616.

3-Cyclohexyl-4,4,4-trifluoro-1-phenylbutan-1-one (2ad) Method A; the product was inseparable from impurities, therefore only the peaks that could be assigned are described.

IR (neat): 3062, 2930, 2856, 1691, 1598, 1581, 1450, 1422, 1390, 1344, 1264, 1240, 1217, 1186, 1154, 1109, 1050, 1019, $1002 \mathrm{~cm}^{-1}$.

${ }^{1} \mathrm{H}$ NMR $\left(\mathrm{CDCl}_{3}\right): \delta=1.07-1.28(\mathrm{~m}, 5 \mathrm{H}), 1.61-1.80(\mathrm{~m}, 6 \mathrm{H})$, $3.06-3.23(\mathrm{~m}, 3 \mathrm{H}), 7.46-7.51(\mathrm{~m}, 2 \mathrm{H}), 7.57-7.61(\mathrm{~m}, 1 \mathrm{H}), 7.97-$ $7.99(\mathrm{~m}, 2 \mathrm{H})$.

${ }^{13} \mathrm{C} \mathrm{NMR}\left(\mathrm{CDCl}_{3}\right): \delta=34.33(\mathrm{q}, J=2.2 \mathrm{~Hz}), 42.87(\mathrm{q}, J=24.6 \mathrm{~Hz})$, 128.37 (q, $J=280.8 \mathrm{~Hz}), 128.08,128.72,133.39,136.52,196.67$.

${ }^{19} \mathrm{~F} \mathrm{NMR}\left(\mathrm{CDCl}_{3}\right): \delta=-67.40(\mathrm{~d}, J=9.8 \mathrm{~Hz}, 3 \mathrm{~F})$.

HRMS: $m / z$ calcd for $\mathrm{C}_{17} \mathrm{H}_{16} \mathrm{~F}_{3} \mathrm{O}(\mathrm{M}+\mathrm{H}): 293.1153$; found: 293.1146 .

\section{4-Methyl-1-phenyl-3-trifluoromethylpentan-1-one (2ae)}

Method B; yield: $38 \mathrm{mg}(0.16 \mathrm{mmol}$, $62 \%)$; white solid; $\mathrm{mp} 28$ $29^{\circ} \mathrm{C}$.

IR (KBr): 2993, 2974, 2947, 2921, 2889, 1684, 1598, 1473, 1452, $1396,1322,1271,1221,1169,1137,1070 \mathrm{~cm}^{-1}$.

${ }^{1} \mathrm{H} \mathrm{NMR}\left(\mathrm{CDCl}_{3}\right): \delta=0.99(\mathrm{~d}, J=7.0 \mathrm{~Hz}, 3 \mathrm{H}), 1.02(\mathrm{~d}, J=7.0 \mathrm{~Hz}$, $3 \mathrm{H}), 2.13$ (dsept, $J=7.0,3.8 \mathrm{~Hz}, 1 \mathrm{H}), 3.02(\mathrm{dd}, J=17.6,5.7 \mathrm{~Hz}$, $1 \mathrm{H}), 3.10-3.18(\mathrm{~m}, 1 \mathrm{H}), 3.22(\mathrm{dd}, J=17.6,5.3 \mathrm{~Hz}, 1 \mathrm{H}), 7.48-7.51$ (m, 2 H), 7.56-7.62 (m, $1 \mathrm{H}), 7.97-8.00(\mathrm{~m}, 2 \mathrm{H})$.

${ }^{13} \mathrm{C} \mathrm{NMR}\left(\mathrm{CDCl}_{3}\right): \delta=19.03,20.20,27.15,33.72(\mathrm{q}, J=2.5 \mathrm{~Hz})$, $42.84(\mathrm{q}, J=24.7 \mathrm{~Hz}), 128.06,128.34(\mathrm{q}, J=281.1 \mathrm{~Hz}), 128.71$, $133.39,136.52,196.62$.

${ }^{19} \mathrm{~F} \mathrm{NMR}\left(\mathrm{CDCl}_{3}\right) ; \delta=-67.96(\mathrm{~d}, J=9.8 \mathrm{~Hz}, 3 \mathrm{~F})$.

HRMS: $m / z$ calcd for $\mathrm{C}_{16} \mathrm{H}_{13} \mathrm{~F}_{3} \mathrm{O}(\mathrm{M}+\mathrm{H})$ : 245.1153; found: 245.1149.

\section{4,4-Dimethyl-1-phenyl-3-trifluoromethylpentan-1-one (2af)}

Method B; the pure product was isolated as a white solid in very low yield; $\mathrm{mp} 39-40{ }^{\circ} \mathrm{C}$.

IR (KBr): 2961, 2880, 1684, 1598, 1478, 1451, 1373, 1355, 1289 , $1265,1202,1147,1096,1074,1028 \mathrm{~cm}^{-1}$.

${ }^{1} \mathrm{H} \mathrm{NMR}\left(\mathrm{CDCl}_{3}\right): \delta=1.06(\mathrm{~s}, 9 \mathrm{H}), 3.02(\mathrm{ddd}, J=18.1,3.9,1.0 \mathrm{~Hz}$, $1 \mathrm{H}), 3.13-3.21$ (ddq, $J=3.9,6.1,10.5 \mathrm{~Hz}, 1 \mathrm{H}), 3.25$ (dd, $J=18.1$, $6.1 \mathrm{~Hz}, 1 \mathrm{H}), 7.46-7.51(\mathrm{~m}, 2 \mathrm{H}), 7.57-7.61(\mathrm{~m}, 1 \mathrm{H}), 7.96-7.99(\mathrm{~m}$, $2 \mathrm{H})$.

${ }^{13} \mathrm{C} \mathrm{NMR}\left(\mathrm{CDCl}_{3}\right): \delta=28.26,32.39,34.89(\mathrm{q}, J=2.8 \mathrm{~Hz}), 46.09(\mathrm{q}$, $J=23.7 \mathrm{~Hz}), 128.51$ (q, $J=282.2 \mathrm{~Hz}), 128.07,128.70,133.36$, $136.49,196.59$.

${ }^{19} \mathrm{~F} \mathrm{NMR}\left(\mathrm{CDCl}_{3}\right): \delta=-64.13(\mathrm{~d}, J=10.5 \mathrm{~Hz}, 3 \mathrm{~F})$.

HRMS: $m / z$ calcd for $\mathrm{C}_{16} \mathrm{H}_{13} \mathrm{~F}_{3} \mathrm{O}(\mathrm{M}+\mathrm{H})$ : 259.1310; found: 259.1314 .

Ethyl 7-Oxa-7-phenyl-5-trifluoromethylheptanoate (2ag)

Method A; the product was inseparable from impurities, and therefore only the peaks that could be assigned are described.

${ }^{19} \mathrm{~F} \mathrm{NMR}\left(\mathrm{CDCl}_{3}\right): \delta=-71.27(\mathrm{~d}, J=7.1 \mathrm{~Hz}, 3 \mathrm{~F})$.

HRMS: $m / z$ calcd for $\mathrm{C}_{16} \mathrm{H}_{20} \mathrm{~F}_{3} \mathrm{O}_{3}(\mathrm{M}+\mathrm{H})$ : 317.1365 ; found: 317.1367 .

\section{7-Ox0-7-phenyl-5-trifluoromethylheptanenitrile (2ah)}

Method A; the product was inseparable from impurities, and therefore only the peaks that could be assigned are described.

${ }^{19} \mathrm{~F} \mathrm{NMR}\left(\mathrm{CDCl}_{3}\right): \delta=-70.96(\mathrm{~d}, J=7.1 \mathrm{~Hz}, 3 \mathrm{~F})$.

HRMS: $m / z$ calcd for $\mathrm{C}_{14} \mathrm{H}_{15} \mathrm{~F}_{3} \mathrm{NO}(\mathrm{M}+\mathrm{H})$ : 270.1106; found: 270.1103 . 


\section{$N, N$-Dibenzyl-3-trifluoromethylpentanamide (2ba)}

Method B; yield: $65 \mathrm{mg}(0.19 \mathrm{mmol}, 74 \%)$; yellow oil.

IR (neat): 3088, 3065, 3031, 2970, 2939, 2883, 1744, 1651, 1606, $1586,1496,1453,1385,1361,1327,1257 \mathrm{~cm}^{-1}$.

${ }^{1} \mathrm{H} \mathrm{NMR}\left(\mathrm{CDCl}_{3}\right): \delta=1.01(\mathrm{t}, J=7.5 \mathrm{~Hz}, 3 \mathrm{H}), 1.21-1.29(\mathrm{~m}, 1 \mathrm{H})$, $1.35-1.40(\mathrm{~m}, 1 \mathrm{H}), 2.43(\mathrm{dd}, J=16.5,7.3 \mathrm{~Hz}, 1 \mathrm{H}), 2.71(\mathrm{dd}, J=$ $16.5,4.8 \mathrm{~Hz}, 1 \mathrm{H}), 2.90-3.10(\mathrm{~m}, 1 \mathrm{H}), 4.44(\mathrm{~d}, J=17.2 \mathrm{~Hz}, 1 \mathrm{H})$, $4.502(\mathrm{~d}, J=14.6 \mathrm{~Hz}, 1 \mathrm{H}), 4.505(\mathrm{~d}, J=17.2 \mathrm{~Hz}, 1 \mathrm{H}), 4.80(\mathrm{~d}, J=$ $14.6 \mathrm{~Hz}, 1 \mathrm{H}), 7.10-7.42(\mathrm{~m}, 10 \mathrm{H})$.

${ }^{13} \mathrm{C} \mathrm{NMR}\left(\mathrm{CDCl}_{3}\right): \delta=11.25,21.70,31.48,40.66(\mathrm{q}, J=25.6 \mathrm{~Hz})$, $48.69,49.77,126.18,127.47$ (q, $J=292.9 \mathrm{~Hz}), 127.51,127.74$, $128.24,128.63,129.04,136.03,137.05,170.46$.

${ }^{19} \mathrm{~F} \mathrm{NMR}\left(\mathrm{CDCl}_{3}\right): \delta=-70.93(\mathrm{~d}, J=7.6 \mathrm{~Hz}, 3 \mathrm{~F})$.

HRMS: $m / z$ calcd for $\mathrm{C}_{20} \mathrm{H}_{22} \mathrm{~F}_{3} \mathrm{NO}(\mathrm{M}+\mathrm{H}): 350.1732$; found: 350.1728 .

\section{2-Trifluoromethylbutyl Phenyl Sulfone (2ca)}

Method B; yield: $45 \mathrm{mg}(0.17 \mathrm{mmol}, 68 \%)$; yellow oil.

IR (neat): 3068, 2977, 2945, 2889, 1586, 1465, 1448, 1414, 1383, $1326,1253,1151,1086,1044,1025 \mathrm{~cm}^{-1}$.

${ }^{1} \mathrm{H}$ NMR $\left(\mathrm{CDCl}_{3}\right): \delta=1.03(\mathrm{dt}, J=7.5,0.81 \mathrm{~Hz}, 3 \mathrm{H}), 1.78-1.88$ (m, $2 \mathrm{H}), 2.69-2.80(\mathrm{~m}, 1 \mathrm{H}), 3.14$ (dd, $J=14.6,8.3 \mathrm{~Hz}, 1 \mathrm{H}), 3.33$ $(\mathrm{dd}, J=14.6,2.9 \mathrm{~Hz}, 1 \mathrm{H}), 7.59-7.62(\mathrm{~m}, 2 \mathrm{H}), 7.68-7.72(\mathrm{~m}, 1 \mathrm{H})$, 7.93-7.95 (m, $2 \mathrm{H})$.

${ }^{13} \mathrm{C} \mathrm{NMR}\left(\mathrm{CDCl}_{3}\right): \delta=10.54,21.32,39.52(\mathrm{q}, J=27.3 \mathrm{~Hz}), 54.00$, 126.81 (q, $J=280.8 \mathrm{~Hz}), 127.93,129.52,134.18,139.02$.

${ }^{19} \mathrm{~F} \mathrm{NMR}\left(\mathrm{CDCl}_{3}\right): \delta=-70.61(\mathrm{~d}, J=7.6 \mathrm{~Hz}, 3 \mathrm{~F})$.

HRMS: $m / z$ calcd for $\mathrm{C}_{14} \mathrm{H}_{18} \mathrm{~F}_{3} \mathrm{O}_{2} \mathrm{~S}(\mathrm{M}+\mathrm{H}): 267.0667$; found: 267.0661 .

\section{Diethyl [2-(Trifluoromethyl)butyl]phosphonate (2da)}

Method B; yield: $45 \mathrm{mg}$ (0.17 mmol, 68\%); yellow oil.

${ }^{1} \mathrm{H} \mathrm{NMR}\left(\mathrm{CDCl}_{3}\right): \delta=1.02(\mathrm{t}, J=7.4 \mathrm{~Hz}, 3 \mathrm{H}), 1.33(\mathrm{t}, J=7.0 \mathrm{~Hz}$, $6 \mathrm{H}), 1.70-1.90(\mathrm{~m}, 3 \mathrm{H}), 2.03$ (ddd, $J=21.2,15.7,3.4 \mathrm{~Hz}, 1 \mathrm{H})$, 2.40-2.55 (m, $1 \mathrm{H}), 4.04-4.17$ (m, $4 \mathrm{H})$.

${ }^{13} \mathrm{C} \mathrm{NMR}\left(\mathrm{CDCl}_{3}\right): \delta=10.68,16.31,16.41,21.60-21.75(\mathrm{~m}, 1 \mathrm{C})$, $24.00(\mathrm{dq}, J=146.2,2.7 \mathrm{~Hz}), 39.32(\mathrm{qd}, J=26.5,2.7 \mathrm{~Hz}), 61.86(\mathrm{~d}$, $J=6.5 \mathrm{~Hz}), 61.95(\mathrm{~d}, J=6.9 \mathrm{~Hz}), 127.77(\mathrm{qd}, J=279.2,18.5 \mathrm{~Hz})$.

${ }^{19} \mathrm{~F} \mathrm{NMR}\left(\mathrm{CDCl}_{3}\right): \delta=-67.40(\mathrm{~d}, J=9.8 \mathrm{~Hz}, 3 \mathrm{~F})$.

HRMS: $m / z$ calcd for $\mathrm{C}_{9} \mathrm{H}_{19} \mathrm{~F}_{3} \mathrm{O}_{3} \mathrm{P}(\mathrm{M}+\mathrm{H})$ : 263.1024; found: 263.1029 .

\section{3-Difluoromethyl-1-phenylpentan-1-one (2ea)}

Method B; yield: $49 \mathrm{mg}(0.21 \mathrm{mmol}, 85 \%)$, yellow oil.

IR (neat) 3062, 2696, 2940, 2882, 1688, 1598, 1581, 1463, 1449, $1380,1359,1320,1260 \mathrm{~cm}^{-1}$.

${ }^{1} \mathrm{H}$ NMR $\left(\mathrm{CDCl}_{3}\right): \delta=0.99(\mathrm{t}, J=7.5 \mathrm{~Hz}, 3 \mathrm{H}), 1.49($ ddq. $J=14.7$, 7.3, 7.3 Hz, $1 \mathrm{H}), 1.65(\mathrm{ddq}, J=14.7,7.4,7.4 \mathrm{~Hz}, 1 \mathrm{H}), 2.53-2.65$ $(\mathrm{m}, 1 \mathrm{H}), 2.99(\mathrm{dd}, J=17.8,6.9 \mathrm{~Hz}, 1 \mathrm{H}), 3.21(\mathrm{dd}, J=17.8,5.6 \mathrm{~Hz}$, $1 \mathrm{H}), 5.94(\mathrm{td}, J=57.5,2.9 \mathrm{~Hz}, 1 \mathrm{H}), 7.45-7.49(\mathrm{~m}, 2 \mathrm{H}), 7.54-7.60$ (m, $1 \mathrm{H}), 7.95-8.00(\mathrm{~m}, 2 \mathrm{H})$.

${ }^{13} \mathrm{C} \mathrm{NMR}\left(\mathrm{CDCl}_{3}\right): \delta=11.33,21.08(\mathrm{dd}, J=5.8,3.5 \mathrm{~Hz}), 35.89(\mathrm{t}$, $J=4.4 \mathrm{~Hz}), 39.39(\mathrm{t}, J=19.1 \mathrm{~Hz}), 117.90(\mathrm{t}, J=241.8 \mathrm{~Hz}), 127.99$, 128.63, 133.25, 136.75, 197.99.

${ }^{19} \mathrm{~F} \mathrm{NMR}\left(\mathrm{CDCl}_{3}\right): \delta=-123.81(\mathrm{ddd}, J=275.3,56.5,14.1 \mathrm{~Hz}, 1 \mathrm{~F})$, -125.17 (ddd, $J=275.3,56.5,19.8 \mathrm{~Hz}, 1 \mathrm{~F}$ ).

HRMS: $m / z$ calcd for $\mathrm{C}_{12} \mathrm{H}_{15} \mathrm{~F}_{2} \mathrm{O}(\mathrm{M}+\mathrm{H})$ : 213.1090; found: 213.1084.

\section{(S)-3-[3-(Trifluoromethyl)pentanoyl]-4-phenyloxazolidin-2-} one (2fa)

Method B.

\section{Major Isomer}

Yield: $45 \mathrm{mg}$ (0.14 mmol, 29\%); white solid; mp 96-97 ${ }^{\circ} \mathrm{C}$.

IR (KBr) 3033, 2974, 2945, 2886, 1786, 1700, 1455, 1414, 1387, $1366,1306,1253,1207,1178,1130,1105,1039 \mathrm{~cm}^{-1}$.

${ }^{1} \mathrm{H} \mathrm{NMR}\left(\mathrm{CDCl}_{3}\right): \delta=0.86(\mathrm{t}, J=7.5 \mathrm{~Hz}, 3 \mathrm{H}), 1.35-1.45(\mathrm{~m}, 1 \mathrm{H})$, $1.62-1.71(\mathrm{~m}, 1 \mathrm{H}), 2.73-2.84(\mathrm{~m}, 1 \mathrm{H}), 3.01(\mathrm{dd}, J=18.3,6.4 \mathrm{~Hz}$, $1 \mathrm{H}), 3.29(\mathrm{dd}, J=18.3,6.0 \mathrm{~Hz}, 1 \mathrm{H}), 4.31(\mathrm{dd}, J=8.9,3.7 \mathrm{~Hz}, 1$ $\mathrm{H}), 4.72(\mathrm{t}, J=8.9 \mathrm{~Hz}, 1 \mathrm{H}), 5.44(\mathrm{dd}, J=8.9,3.7 \mathrm{~Hz}, 1 \mathrm{H}), 7.29$ $7.31(\mathrm{~m}, 2 \mathrm{H})$, 7.33-7.41 (m, $3 \mathrm{H})$.

${ }^{13} \mathrm{C} \mathrm{NMR}\left(\mathrm{CDCl}_{3}\right): \delta=10.98,21.42(\mathrm{q}, J=2.3 \mathrm{~Hz}), 33.96(\mathrm{q}, J=$ $2.5 \mathrm{~Hz}), 39.66(\mathrm{q}, J=26.0 \mathrm{~Hz}), 57.76,70.11,125.90,127.86(\mathrm{q}, J=$ $279.8 \mathrm{~Hz}), 128.86,129.21,138.72,153.61,169.96$.

${ }^{19} \mathrm{~F} \mathrm{NMR}\left(\mathrm{CDCl}_{3}\right): \delta=-71.30(\mathrm{~d}, J=9.8 \mathrm{~Hz}, 3 \mathrm{~F})$.

HRMS: $m / z$ calcd for $\mathrm{C}_{15} \mathrm{H}_{17} \mathrm{~F}_{3} \mathrm{NO}_{3}(\mathrm{M}+\mathrm{H}): 316.1161$; found: 316.1154 .

\section{Minor Isomer}

Yield: $22 \mathrm{mg}$ (0.07 mmol, 14\%); white solid; $\mathrm{mp} 54-55^{\circ} \mathrm{C}$.

IR (KBr) 3068, 3035, 2985, 2946, 2884, 1790, 1697, 1473, 1395, $1336,1232,1208,1169,1139,1124,1084,1069,1045,1015 \mathrm{~cm}^{-1}$.

${ }^{1} \mathrm{H} \mathrm{NMR}\left(\mathrm{CDCl}_{3}\right): \delta=0.97(\mathrm{t}, J=7.4 \mathrm{~Hz}, 3 \mathrm{H}), 1.44-1.50(\mathrm{~m}, 1 \mathrm{H})$, $1.67-1.72(\mathrm{~m}, 1 \mathrm{H}), 2.70-2.81(\mathrm{~m}, 1 \mathrm{H}), 3.05(\mathrm{dd}, J=18.2,7.2 \mathrm{~Hz}$, $1 \mathrm{H}), 3.27(\mathrm{dd}, J=18.2,5.3 \mathrm{~Hz}, 1 \mathrm{H}), 4.30(\mathrm{dd}, J=8.9,4.0 \mathrm{~Hz}, 1$ H), $4.72(\mathrm{t}, J=8.9 \mathrm{~Hz}, 1 \mathrm{H}), 5.43(\mathrm{dd}, J=8.9,4.0 \mathrm{~Hz}, 1 \mathrm{H}), 7.27-$ 7.30 (m, $2 \mathrm{H}), 7.33-7.41$ (m, $3 \mathrm{H})$.

${ }^{13} \mathrm{C} \mathrm{NMR}\left(\mathrm{CDCl}_{3}\right): \delta=11.20,21.44(\mathrm{q}, J=2.0 \mathrm{~Hz}), 34.13(\mathrm{q}, J=$ $2.6 \mathrm{~Hz}), 39.70(\mathrm{q}, J=26.0 \mathrm{~Hz}), 57.81,70.11,125.81,127.83(\mathrm{q}, J=$ $278.2 \mathrm{~Hz}), 128.89,129.25,138.54,153.59,170.00$.

${ }^{19} \mathrm{~F} \mathrm{NMR}\left(\mathrm{CDCl}_{3}\right): \delta=-71.22(\mathrm{~d}, J=7.5 \mathrm{~Hz}, 3 \mathrm{~F})$.

HRMS: $m / z$ calcd for $\mathrm{C}_{15} \mathrm{H}_{17} \mathrm{~F}_{3} \mathrm{NO}_{3}(\mathrm{M}+\mathrm{H}): 316.1161$; found: 316.1154 .

\section{(S)-3-[3-(Trifluoromethyl)pentanoyl]-4-benzyloxazolidin-2-one (2ga)} Method B

\section{Major Isomer}

Yield: $35 \mathrm{mg}(0.11 \mathrm{mmol}, 31 \%)$; yellow oil.

IR (neat): 3088, 3065, 3030, 2974, 2944, 2886, 1778, 1701, 1605, $1498,1482,1455,1390,1327,1257,1212,1169,1134,1049,1030$ $\mathrm{cm}^{-1}$.

${ }^{1} \mathrm{H} \mathrm{NMR}\left(\mathrm{CDCl}_{3}\right): \delta=1.04(\mathrm{t}, J=7.5 \mathrm{~Hz}, 3 \mathrm{H}), 1.49-1.58(\mathrm{~m}, 1 \mathrm{H})$, $1.75-1.84(\mathrm{~m}, 1 \mathrm{H}), 2.76(\mathrm{dd}, J=13.4,9.7 \mathrm{~Hz}, 1 \mathrm{H}), 2.86-2.95(\mathrm{~m}$, $1 \mathrm{H}), 3.08$ (dd, $J=18.1,6.3 \mathrm{~Hz}, 1 \mathrm{H}), 3.23(\mathrm{dd}, J=18.1,6.1 \mathrm{~Hz}, 1$ H), $3.31(\mathrm{dd}, J=13.4,3.4 \mathrm{~Hz}, 1 \mathrm{H}), 4.19(\mathrm{dd}, J=9.1,2.9 \mathrm{~Hz}, 1 \mathrm{H})$, $4.23(\mathrm{dd}, J=9.1,7.7 \mathrm{~Hz}, 2 \mathrm{H}), 4.67-4.72(\mathrm{~m}, 1 \mathrm{H}), 7.20-7.22(\mathrm{~m}, 2$ H), 7.26-7.30 (m, $1 \mathrm{H}), 7.31-7.36(\mathrm{~m}, 2 \mathrm{H})$.

${ }^{13} \mathrm{C} \mathrm{NMR}\left(\mathrm{CDCl}_{3}\right): \delta=11.19,21.41(\mathrm{q}, J=2.4 \mathrm{~Hz}), 34.00(\mathrm{q}, J=$ $2.2 \mathrm{~Hz}$ ), 37.82, 39.77 (q, $J=26.2 \mathrm{~Hz}), 55.29,66.40,127.42,127.91$ $(\mathrm{q}, J=279.8 \mathrm{~Hz}), 128.98,129.34,135.02,153.34,170.42$.

${ }^{19} \mathrm{~F} \mathrm{NMR}\left(\mathrm{CDCl}_{3}\right): \delta=-71.19(\mathrm{~d}, J=9.8 \mathrm{~Hz}, 3 \mathrm{~F})$.

HRMS: $m / z$ calcd for $\mathrm{C}_{16} \mathrm{H}_{19} \mathrm{~F}_{3} \mathrm{NO}_{3}(\mathrm{M}+\mathrm{H}): 330.1317$; found: 330.1309 .

\section{Minor Isomer}

Yield: $30 \mathrm{mg}(0.09 \mathrm{mmol}, 27 \%)$; yellow oil.

IR (neat): 3029, 2967, 2943, 2881, 1786, 1703, 1455, 1391, 1257, $1213,1170,1134,1104,1030 \mathrm{~cm}^{-1}$.

${ }^{1} \mathrm{H} \mathrm{NMR}\left(\mathrm{CDCl}_{3}\right): \delta=1.02(\mathrm{t}, J=7.5 \mathrm{~Hz}, 3 \mathrm{H}), 1.48-1.57(\mathrm{~m}, 1 \mathrm{H})$, $1.73-1.81(\mathrm{~m}, 1 \mathrm{H}), 2.77(\mathrm{dd}, J=13.3,9.6 \mathrm{~Hz}, 1 \mathrm{H}), 2.87-2.96(\mathrm{~m}$, $1 \mathrm{H}), 2.99(\mathrm{dd}, J=18.1,6.5 \mathrm{~Hz}, 1 \mathrm{H}), 3.31(\mathrm{dd}, J=13.3,3.3 \mathrm{~Hz}, 1$ H), $3.33(\mathrm{dd}, J=18.1,5.6 \mathrm{~Hz}, 1 \mathrm{H}), 4.20(\mathrm{dd}, J=9.1,3.2 \mathrm{~Hz}, 1 \mathrm{H})$, $4.23(\mathrm{dd}, J=9.1,7.6 \mathrm{~Hz}, 1 \mathrm{H}), 4.67-4.71(\mathrm{~m}, 1 \mathrm{H}), 7.19-7.22(\mathrm{~m}, 2$ H), 7.26-7.30 (m, $1 \mathrm{H}), 7.33-7.35(\mathrm{~m}, 2 \mathrm{H})$. 
${ }^{13} \mathrm{C} \mathrm{NMR}\left(\mathrm{CDCl}_{3}\right): \delta=11.24,21.54(\mathrm{q}, J=2.0 \mathrm{~Hz}), 34.04(\mathrm{q}, J=$ $2.5 \mathrm{~Hz}), 37.69,39.78$ (q, $J=26.1 \mathrm{~Hz}), 55.27,66.33,127.42,127.94$ (q, $J=279.8 \mathrm{~Hz}), 129.00,129.35,134.98,153.31,170.48$.

${ }^{19} \mathrm{~F} \mathrm{NMR}\left(\mathrm{CDCl}_{3}\right): \delta=-70.17(\mathrm{~d}, J=9.8 \mathrm{~Hz}, 3 \mathrm{~F})$.

HRMS: $m / z$ calcd for $\mathrm{C}_{16} \mathrm{H}_{19} \mathrm{~F}_{3} \mathrm{NO}_{3}(\mathrm{M}+\mathrm{H})$ : 330.1317; found: 330.1325 .

Supporting Information for this article is available online at http://www.thieme-connect.com/ejournals/toc/synthesis.

\section{References}

(1) (a) Perlmutter, O. Conjugate Addition Reactions in Organic Synthesis; Pergamon Press: Oxford, 1992. (b) Jung, M. E. In Comprehensive Organic Synthesis; Vol. 4; Trost, B. M.; Fleming, I., Eds.; Pergamon: Oxford, 1991, 1-67.

(2) For selected reviews on asymmetric 1,4-conjugate addition, see: (a) Alexakis, A.; Bäckvall, J. E.; Krause, N.; Pámies, O.; Diéguez, M. Chem. Rev. 2008, 108, 2796. (b) Harutyunyan, S. R.; Hartog, T. D.; Geurt, K.; Minnaard, A. J.; Feringa, B. L. Chem. Rev. 2008, 108, 2824. (c) Hayashi, T. Bull. Chem. Soc. Jpn. 2004, 77, 13. (d) Hayashi, T.; Yamasaki, K. Chem. Rev. 2003, 103, 2829. (e) Alexakis, A.; Belhaim, C. Eur. J. Org. Chem. 2002, 3221. (f) Hayashi, T. Synlett 2001, 879. (g) Krause, N.; Hoffmann-Röder, A. Synthesis 2001, 171. (h) Sibi, M.; Manyem, S. Tetrahedron 2000, 56, 8033. (i) Leonard, J.; Diez-Barra, E.; Merino, S. Eur. J. Org. Chem. 1998, 2051. (j) Rossiter, B. E.; Swingle, N. M. Chem. Rev. 1992, 92, 771.

(3) General reviews on organozinc reagents: (a) Knochel, P.; Millot, N.; Rodrigues, A. L.; Tucker, C. E. Org. React. 2001, 58, 417. (b) Knochel, P.; Jones, P. Organozinc Reagents; Oxford University Press: Oxford, 1999. (c) Knochel, P.; Singer, R. D. Chem. Rev. 1993, 93, 2117. For recent studies on organozinc reagents, see: (d) Endo, K.; Hamada, D.; Yakeishi, S.; Ogawa, M.; Shibata, T. Org. Lett. 2012, 14, 2342. (e) Lemaire, S.; Houpis, I. N.; Xiao, T.; Li, J.; Digard, E.; Gozlan, C.; Liu, R.; Gavryushin, A.; Diène, C.; Wang, Y.; Farina, V.; Knochel, P. Org. Lett. 2012, 14, 1480.

(4) For comprehensive reviews on diorganozinc reagents, see: (a) Lemire, A.; Côté, A.; Janes, M. K.; Charette, A. B. Aldrichimica Acta 2009, 42, 71. (b) Hanson, M. V.; Rieke, R. D. J. Am. Chem. Soc. 1995, 117, 10775. (c) Knochel, P.; Perea, J. J. A.; Jones, P. Tetrahedron 1998, 54, 8275.

(5) (a) Uneyama, K. Organofluorine Chemistry; Blackwell: Oxford, 2006. (b) Chambers, R. D. Fluorine in Organic Chemistry; Blackwell: Oxford, 2004. (c) Kirsch, P. Modern Fluoroorganic Chemistry; Wiley-VCH: Weinheim, 2004.

(6) Shinohara, N.; Haga, J.; Yamazaki, T.; Kitazume, T.; Nakamura, S. J. Org. Chem. 1995, 60, 4363.

(7) (a) Konno, T.; Tanaka, T.; Miyabe, T.; Morigaki, A.; Ishihara, T. Tetrahedron Lett. 2008, 49, 2106. (b) Yamazaki, T.; Shinohara, N.; Kitazume, T.; Sato, S. J. Fluorine Chem. 1999, 97, 91; and references cited therein.

(8) The alkylzinc halides, RZnI, were prepared according to the reported procedure; see ref. $4 \mathrm{~b}$.

(9) The prepared dialkylzinc reagents were used without the removal of $\mathrm{LiCl}$. For the detailed preparation procedure, see the experimental section.

(10) The lithium zincate, $(n-\mathrm{Bu})_{3} \mathrm{ZnLi}$, was prepared by mixing 3 equiv of $n-\mathrm{BuLi}$ (1.6 M hexane solution) and 1 equiv of $\mathrm{ZnCl}_{2}\left(1.0 \mathrm{M}, \mathrm{Et}_{2} \mathrm{O}\right.$ solution) at $0{ }^{\circ} \mathrm{C}$ for $30 \mathrm{~min}$.

(11) The reactivity of dialkylzinc reagents is: salt-free $\mathrm{R}_{2} \mathrm{Zn}>$ in situ generated $R_{2} Z n$.

(12) The control experiments were conducted as follows. The 1,4-conjugated additions to $(E)$-1-phenylbut-2-en-1-one or chalcone with $\mathrm{Et}_{2} \mathrm{Zn}$ were attempted under the same conditions as given in Table 1, entry 2; however, a quantitative amount of the starting alkene was recovered in both cases (methods A and B). 